\section{MORTALIDAD DE MÉDICOS QUE REALIZAN EL SERVICIO RURAL (SERUMS) EN PERÚ, 2006-2009}

\section{[MORTALITY IN YOUNG DOCTORS DOING THE RURAL SERVICE IN PERU, 2006-2009]}

\author{
Edén Galán-Rodas ${ }^{1, a}$, Cristian Díaz-Vélez ${ }^{1, b}$, \\ Juan Villena ${ }^{2, c}$, Ciro Maguiña ${ }^{3, c}$
}

Sr. Editor. En el último número de su revista, Choquehuanca-Vilca et al. (1) mencionan que en las muertes por accidente de tránsito el grupo de edad más afectado resultó ser el comprendido entre 24 a 34 años, grupo que se encuentra dentro de la población económicamente activa, que incluye a los profesionales más jóvenes. Si bien es cierto que en el artículo no se menciona este aspecto, consideramos importante hacer referencia sobre la mortalidad de la profesión médica en sus recién egresados, sobre todo, por darse en pleno cumplimiento de sus funciones.

En el año 1975, se crea el Servicio Civil de Graduandos (SECIGRA), posteriormente, en el año 1981, nace el Servicio Rural Urbano Marginal en Salud (SERUMS) como primera experiencia profesional para los médicos recién egresados, el cual se realiza en distintos ámbitos geográficos de Perú, permitiendo no solo la aplicación de nuestros conocimientos en favor de las poblaciones objetivo, sino también representar al Estado Peruano de acuerdo con la Ley General de Salud N. ${ }^{\circ} 26842$, que establece: La salud es condición indispensable del desarrollo humano y medio fundamental para alcanzar el bienestar individual y colectivo; así también: La protección de la salud es de interés público. Por tanto, es responsabilidad del Estado regularla, vigilarla y promoverla.

Durante el SERUMS, muchos encontramos realidades frustrantes, no solo por las difíciles condiciones geográficas o por la carencia de condiciones mínimas en infraestructura e insumos para el ejercicio profesional, sino también por la creciente inseguridad a la que se expone el médico - maltrato, violaciones, acoso, entre otras -, que se evidencia por la muerte de los profesionales médicos ${ }^{(2)}$, hechos que no se convierten en noticia ni es muy divulgada por las

Comité Médico Joven, Colegio Médico del Perú. Lima, Perú.

2 Secretario del Interior, Colegio Médico del Perú. Lima, Perú.

3 Decanato, Colegio Médico del Perú. Lima, Perú.

a Médico; b Médico epidemiólogo; ' Médico infectólogo.

Recibido: 23-08-10 Aprobado: 01-09-10 entidades correspondientes o por los medios de comunicación. Por ello, tuvimos como objetivo conocer la tasa de mortalidad de los médicos que realizan el SERUMS y las causas de muerte en el periodo 20062009.

En ese sentido, realizamos un análisis de fuentes secundarias, se usó la base de datos del Colegio Médico del Perú (CMP) y el reporte de Seguros MAPFRE para el registro de muertes de los profesionales médicos a nivel nacional; el número de plazas adjudicadas en cada año fue obtenida de la Oficina de Recursos Humanos del Ministerio de Salud.

Se halló diez fallecidos durante los años en evaluación, sus edades oscilaron entre 24 y 33 años, y la proporción en cuanto a sexo fue de $5 / 5$, la mayoría de ellos eran colegiados en el Consejo Regional III - Lima y procedentes de universidades de la capital (7/10), la causa predominante de muerte fue por accidentes de tránsito (7/10), seguido del suicidio $(2 / 10)$ y asfixia por ahogamiento $(1 / 10)$.

La tasa de mortalidad se calculó teniendo como base a los fallecidos según el número de plazas adjudicadas en cada año, se encontró en los últimos dos años un aumento en esta tasa, que llega a ser más de dos médicos por cada mil plazas adjudicadas (Figura 1).

Los accidentes de tránsito son la causa principal de la mortalidad en médicos que realizan el SERUMS, considerando que forman parte del grupo de edad más afectado $^{(1)}$; sin embargo, cabe resaltar que en los estudios

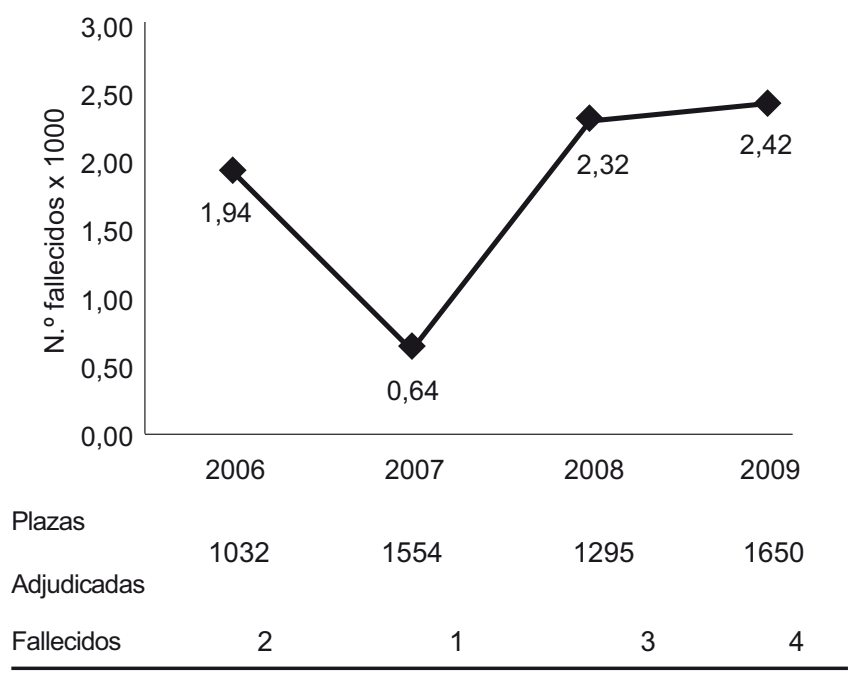

Figura 1. Tasa de mortalidad de los médicos que realizan el SERUMS, Perú 2006-2009. 
revisados, las características de la mortalidad de los médicos en el mundo, están principalmente atribuidas a la longevidad con enfermedades crónicas de predominio cardiovascular, pero ningún estudio refiere mortalidad en el primer año de ejercicio profesional (3-5) y sólo en Malawi - África se reporta una muerte por accidente de tránsito en un periodo de cinco años después de egresar (6). Por otro lado, el suicidio representa la segunda causa de mortalidad, con predominio del sexo femenino, dato similar al de otras latitudes ${ }^{(3)}$.

Finalmente, resaltar que si bien en los últimos años el número de plazas SERUMS para los profesionales de la salud se han incrementado, esto viene acompañado de riesgo de muerte ${ }^{(2)}$, el que se evidencia por las cifras antes mencionadas y que responde a un inadecuado funcionamiento del transporte público, carencia de campañas de concientización a la población y la falta de articulación entre los poderes del Estado para la toma de decisiones.

El acto médico es complejo y está supeditado a muchas condiciones, el médico recién egresado que va a la comunidad realiza este acto con gran sacrificio y dedicación, e incluso arriesgando su vida tal como ocurrió con los casos mencionados. Es pues, necesario actuar con mayor seriedad y eficiencia ante esta lamentable realidad, pues no es razonable que la vida de quienes tienen como objetivo principal salvar vidas, carezca de condiciones mínimas en su integridad y seguridad. El Colegio Médico del Perú es quien asume desde el año 2006 la responsabilidad de brindar el seguro de vida contra accidentes ante una falta de cobertura del Estado, a quienes les corresponde por ser sus empleadores.

\section{Conflictos de Interés}

Los autores declaran no tener conflictos de interés en la publicación de este artículo.

\section{REFERENCIAS BIBLIOGRÁFICAS}

1. Choquehuanca-Vilca V, Cardenas-Garcia F, CollazosCarhuay J, Mendoza-Valladolid W. Perfil epidemiológico de los accidentes de tránsito en el Perú, 2005-2009. Rev Peru Med Exp Salud Publica. 2010;27(2):162-9.

2. Wong P. Los nuevos mártires de la medicina en el Perú. An Fac Med (Lima). 2009;70(2):151-52.

3. Frank E, Biola $\mathbf{H}$, Burnett C. Mortality rates and causes among U.S. physicians. Am J Prev Med. 2000;19(3):155-59.

4. Schernhammer E, Colditz GA. Suicide rates among physicians: a quantitative and gender assessment (metaanalysis). Am J Psychiatry. 2004;161(12):2295-302.

5. Ciriacos $\mathbf{C}$, Rodríguez $\mathbf{H}$, Turnes $\mathbf{A}$. Mortalidad de los médicos en Uruguay (segunda parte). Análisis de las causas de muerte en el quinquenio 1998-2002. Rev Med Urug. 2006;22(4):277-86.
6. Muula AS. Country of residence and deaths among medical graduates from the Malawi College of Medicine, $1992-$ 2002. S Afr Med J. 2006,96(8):662.

Correspondencia: Edén Galán Rodas

Dirección: Av. Malecón Armendáriz 791, Lima 18, Perú.

Correo electrónico: edgarod6@gmail.com

\section{ACCIDENTES DE TRÁNSITO EN LA SELVA: OTRAS RAZONES PARA DISCUTIR}

\section{[ROAD TRAFFIC INJURIES IN THE JUNGLE: OTHER REASONS TO DISCUSS]}

\author{
Charles Huamaní1,3,a; Ysel Cabrera ${ }^{2,3, a}$
}

Sr. Editor. En el reciente número de su Revista dedicada a los accidentes de tránsito se ha dejado al descubierto la gran deficiencia de los registros tradicionales de accidentes de tránsito, en especial al contrastar las cifras obtenidas de la estadística de la Policía Nacional del Perú (PNP) ${ }^{(1)}$ frente a los valores autorreportados en una encuesta nacional (2); pues al observar la distribución geográfica de los accidentes, según la estadística de la PNP el 63,7\% de accidentes suceden en Lima en contraste con el mayor riesgo de accidentes que presentan los habitantes de la selva (OR: 2,03$)^{(2)}$.

Si bien la estadística de la PNP es la más empleada en diversos estudios, su constitución no se debe a métodos rigurosos ni sistemáticos ${ }^{(3)}$, y tal como lo grafican Wong et al., existe una disociación entre lo informado por la PNP y lo identificado por los servicios de salud. Tampoco se puede señalar que lo medido en esa encuesta sea exacto, toda vez que esta no tuvo como objetivo principal determinar el número de accidentes de tránsito, pero nos da luces de un fenómeno que no está siendo bien estudiado.

Según el estudio de Wong et al. (2) la región San Martín tiene un 3,8\% de autorreporte de accidentes de tránsito, mayor que en Lima o Arequipa (de forma curiosa, Arequipa tiene el mayor número de accidentes de tránsito según la estadística de la PNP, pero el menor porcentaje de autorreporte de accidentes según la encuesta de DEVIDA). Esta diferencia puede deberse a que las posibles causas de accidentes de tránsito difieren a las señaladas por Choquehuanca et al. ${ }^{(1)}$.

\footnotetext{
Sociedad Científica de San Fernando, Facultad de Medicina, Universidad Nacional Mayor de San Marcos. Lima, Perú.

2 Facultad de Medicina, Universidad San Martin de Porres. Lima, Perú.

3 Centro de Salud Naranjos, Dirección Regional de Salud San Martín, Gobierno Regional San Martín. San Martin, Perú.

a Médico cirujano.
}

Recibido: 24-08-10 Aprobado: 01-09-10 